Artículo Original/ Original Article

http://dx.doi.org/10.18004/mem.iics/1812-9528/2021.019.02.6

\title{
Determinantes sociales de la mortalidad infantil reducible en departamentos del noroeste argentino entre los años 2010 y 2014
}

\author{
Carola Leticia Bertone ${ }^{1}{ }^{1,2}$, Marcos Javier Andrada $(D)$, , Víctor Eduardo \\ Torres (iD) 1,3 \\ ${ }^{1}$ Consejo Nacional de Investigaciones Científicas y Técnicas (CONICET). \\ Buenos Aires, Argentina \\ ${ }^{2}$ Universidad Nacional de La Rioja, Argentina \\ ${ }^{3}$ Universidad Nacional de Córdoba, Facultad de Ciencias Económicas. Córdoba, \\ Argentina
}

Cómo referenciar este artículo/ How to reference this article:
Bertone CL, Andrada MJ, Torres VE. Determinantes sociales de la mortalidad infantil reducible en departamentos del noroeste argentino. Mem. Inst. Investig. Cienc. Salud. 2021; 19(2): 6-20

\begin{abstract}
RES U M E N
Este artículo compara los determinantes sociales de la salud (DSS) entre los departamentos del Noroeste Argentino que poseen alto riesgo de mortalidad infantil neonatal o pos-neonatal reducible y el resto de ellos, entre los años 2010 y 2014 . El análisis de las condiciones socioeconómicas y su vínculo con la mortalidad infantil aporta conocimiento necesario respecto a la importancia de la consideración del entorno de las poblaciones y resulta imprescindible para formular políticas públicas adecuadas. Se propone un estudio ecológico con datos censales de 2010 y estadísticas vitales. Se identifican los departamentos de alto riesgo de muerte mediante el software libre SaTScan. Luego, se realiza una prueba $\mathrm{T}$ de comparación de medias para muestras independientes. De esta forma, se identificaron mayores deficiencias en los DSS de las zonas con alto riesgo.
\end{abstract}

Palabras clave: análisis espacial, determinantes sociales de la salud, mortalidad infantil.

\section{Social determinants of infant mortality due to reducible causes in counties of northwest of Argentina}

\begin{abstract}
A B S T R A C T
We aim to compare the social determinants of health (DSS) between the departments of the Argentine Northwest with a high risk of reducible neonatal and postneonatal infant mortality and the rest of them, between 2010 and 2014.

Consideration of socioeconomic conditions and their link with infant mortality provides essential knowledge regarding the importance of considering the environment of populations and is essential for formulating adequate public policies.

An ecological study is proposed, with census data from 2010, and vital statistics. Departments at high risk of death are identified using free Satscan software, and then a mean comparison t-test is performed for independent samples. Greater deficiencies were identified in the DSS in high-risk areas.
\end{abstract}

Keywords: infant mortality, social determinants of health, spatial analysis.

Fecha de recepción: Abril 2021. Fecha de aceptación: agosto 2021

*Autor correspondiente: Carola Leticia Bertone. Consejo Nacional de Investigaciones Científicas y Técnicas (CONICET). Buenos Aires, Argentina

Email: marcosandrada80@gmail.com 


\section{INTRODUCCIÓN}

Autores reconocidos en la temática de la mortalidad infantil (MI), tales como como Finkelstein, et al. ${ }^{(1)}$ y Mazzeo ${ }^{(2)}$ afirman que Argentina muestra un patrón de descenso sostenido de este indicador, alcanzando en el año 2016 los 9,7 muertos por cada 1000 nacidos vivos; sin embargo, las desigualdades al interior del país aún persisten. El noroeste argentino (NOA) es reconocido desde hace años como área prioritaria por el Estado para la aplicación de las políticas públicas en la reducción de la MI. Las muertes infantiles pasibles de ser reducidas representan el $60 \%$ actualmente en el país. Se entiende por mortalidad infantil a aquella que ocurre antes del primer aniversario de vida.

Este artículo se posiciona desde la perspectiva de los Determinantes Sociales de la Salud (DSS). En ella se sostiene que:

Las condiciones de salud de una población son el resultado del efecto conjunto de factores genéticos, estilos de vida y factores del contexto, donde desarrolla su vida. Es decir, la situación de la salud y sus desigualdades están determinadas tanto individual como histórica y socialmente. Si bien los factores genéticos pueden tener un peso relevante en la salud individual, a nivel de la población, son los factores socioeconómicos, culturales, ambientales y políticos los que tienen el rol fundamental como determinantes de los niveles de salud ${ }^{(3)}$.

En Argentina, la primera década del año 2000 se caracterizó por un fuerte crecimiento económico y una significativa recomposición de los indicadores sociales después de la crisis que se vivió en 2001 y 2002. No obstante, se dio un aumento en las disparidades inter-provinciales en relación tanto a las desigualdades sociales como económicas. Al respecto, las provincias rezagadas (en su mayoría, las del norte del país) cosecharon mayores avances relativos, lo que refuerza lo mencionado por Bolsi, Longhi y Paolasso ${ }^{(4)}$ cuando sostienen que el Norte Argentino es la región más pobre del país y se mantiene así desde hace décadas.

Hay gran variedad de trabajos que explican la relación existente entre las condiciones sociales desfavorables y los determinantes sociales de mortalidad, aunque a veces no son estrictamente definidos como tales ${ }^{(5)}$. Algunos de los trabajos reconocidos en los países desarrollados son los propuestos por Wagstaff( ${ }^{(6)}$, mientras que en Latinoamérica puede rescatarse los aportes de Behm $^{(7)}$, Benavides ${ }^{\left({ }^{(1)}\right.}$ y López Pardo ${ }^{(9)}$. Por su parte, y particularmente en Argentina se identifican los aportes de Etchegoyen y Paganini ${ }^{(10)}$; Buchbinder ${ }^{(11)}$; Bolsi, Longhi, y Paolasso ${ }^{(4)}$, Longhi ${ }^{(12)}$ y Terrasa, Rubinstein, Giraudo, y Bortman ${ }^{(13)}$.

Este artículo se realiza exclusivamente sobre el Noroeste Argentino (NOA) ${ }^{1}$, que es una región que todavía puede disminuir su MI. A fin de poner en contexto su situación, puede decirse que entre 1994 y 2003 el $41 \%$ de los departamentos del NOA exhibieron una tendencia marcadamente decreciente en cuanto a la $\mathrm{MI}^{(6)}$. En el año 2008, alguno de esos departamentos presentaron tasas de MI superiores a $40 \%$ o nacidos vivos ${ }^{(7)}$. Por su parte, Bertone ${ }^{(8)}$, quien estudió la MI y sus determinantes en Argentina entre 2000 y 2010 , detectó que varios departamentos del NOA constituían zonas de riesgo de mortalidad neonatal y pos-neonatal, donde existían condiciones de carencia respecto a ciertos determinantes intermediarios. Otro estudio, el de Herrero y Bossio $^{(9)}$, asegura que las desigualdades sociales y las inequidades influyen en la MI reducible. En el mismo se consideran los efectos de las características individuales, de las cualidades grupales de donde provienen y de los departamentos donde residen los niños, señalando que a su vez estos efectos inciden entre sí.

Teniendo en cuenta esto, el objetivo que se plantea este artículo es cotejar algunos determinantes sociales intermediarios entre departamentos del Noroeste Argentino (NOA), comparando aquellos identificados como áreas críticas o de mayor riesgo de morir durante el primer año de vida por causas de muerte reducibles y el resto de la región, empleando para ello el período comprendido entre los años 2010 y 2014.

En 2005 se creó la Comisión sobre los Determinantes Sociales de la Salud (CDSS) en la Organización Mundial de la Salud (OMS) que tiene como objetivo fundamental elaborar el marco conceptual en el que se pone en relieve la diferencia entre los

\footnotetext{
${ }^{1}$ El Noroeste Argentino se conforma por las provincias de Catamarca, Jujuy, La Rioja, Salta, Santiago del Estero y Tucumán.
} 
niveles de causalidad, la distinción entre los mecanismos por los cuales se crean las jerarquías sociales y resultan las condiciones de la vida cotidiana ${ }^{(10)}$. Esto se hace por medio de la sistematización y el resumen de las evidencias sobre cómo la estructura de las sociedades afecta la salud de la población y analiza las estrategias que los gobiernos y la salud pública pueden llevar a cabo para mejorar la salud de la población. Se llamó, entonces, determinantes sociales de la salud a todas aquellas circunstancias materiales psicosociales, factores conductuales y/o biológicos, definidos por los mecanismos sociales, económicos y políticos, que dan lugar a un conjunto de posiciones socioeconómicas, a partir de las cuales las poblaciones están estratificadas de acuerdo a ingresos, educación, ocupación, género, raza/origen étnico, entre otros factores ${ }^{(10)}$.

Previamente, se había desarrollado un marco conceptual específicamente referido a la niñez, elaborado por Irwin, Siddiqi y $\operatorname{Hertzman}^{(11)}$ y se lo propuso para enmarcar los tipos de entornos $-y$, por tanto, las experiencias- que son integrales. A su vez, se los debe vincular con los procesos biológicos con los cuales interactúan para determinar los resultados de la niñez. Se trata de esferas concéntricas, cuyo centro es el individuo y hacia afuera se encuentra la familia y la vivienda, las comunidades residenciales y relacionales, los programas y servicios para el Desarrollo de la Primera Infancia (DPI) y los ámbitos regionales, nacionales y mundiales. Cada una de estas esferas -que no son jerárquicas, sino que están interrelacionados-incluyen factores sociales, económicos, culturales y de género que inciden en sus cualidades enriquecedoras ${ }^{(11)}$.

El enfoque de los determinantes de la salud ha ido evolucionando a lo largo del tiempo desde la década de 1990, dando lugar a múltiples modelos que buscan explicar la influencia de éstos en la salud de las poblaciones y en las desigualdades de salud que se observan entre diferentes grupos sociales.

\section{MATERIAL Y MÉTODOS}

En el presente artículo se realiza un estudio observacional transversal en el cual se emplea como fuente de datos el censo de Población, Hogares y Viviendas del 2010 (INDEC) como así también las bases de datos de los registros de nacimientos y defunciones infantiles entre el año 2010 y 2014 del Ministerio de Salud de la Nación.

Cabe aclarar que se trata de un estudio ecológico, entendiendo como tal a aquel en el que las unidades de análisis son poblaciones, subpoblaciones o grupos de personas $^{(17)}$.

Distintos estudios teóricos y empíricos demostraron diferencias significativas en las condiciones de salud entre los diversos grupos sociales definidos según sus ingresos, educación, origen étnico, género, área de residencia o por factores ambientales. Dicha desigualdad compromete a los sectores más desfavorecidos ayudando a la falta de cohesión social e impactando profundamente en la situación de la infancia ${ }^{(3)}$.

De este modo, la condición de la vivienda, y ciertas características del hogar (hacinamiento, contaminación del aire en el interior de la misma, humedad y frío) se relaciona con la salud física y mental ${ }^{(11)}$ e influye en la calidad de atención y cuidado que se les brinda a los niños.

Existen evidencias que incluso si la privación de servicios básicos fuera moderada, también contribuyen de manera significativa para que la pobreza infantil aumente considerablemente. A propósito de ello, se incluyó el porcentaje de viviendas en zonas rurales dispersas porque los niños de estas zonas (en su mayoría) están privados de varias necesidades que constituyen sus derechos fundamentales. Entre ellos está el servicio de red eléctrica, ya que se considera indispensable para poder llegar a acceder y utilizar las nuevas tecnologías de la información y las comunicaciones que son en la actualidad instrumentos cruciales para la formación del capital humano ${ }^{(12)}$.

También se incorporaron algunas variables incluidas en el método de caracterización de la pobreza, como son las Necesidades Básicas Insatisfechas ${ }^{2}$ que

\footnotetext{
${ }^{2}$ Las Necesidades Básicas Insatisfechas (NBI) fueron definidas según la metodología utilizada en La pobreza en la Argentina (serie Estudios INDEC, $N^{\circ} 1$, Buenos Aires, 1984). Un hogar con NBI es aquel que presenta al menos uno de los siguientes indicadores de privación:

A. Hacinamiento: más de tres personas por cuarto.

B. Vivienda: habitan en una vivienda de tipo inconveniente (pieza de inquilinato, pieza de hotel o pensión, casilla, local no
} 
hacen referencia a las condiciones sanitarias del hogar, al hacinamiento y a la subsistencia. Sin embargo, no se pudo incluir a algunos aspectos asociados con el bienestar de las madres, refiriéndose particularmente a aquellas mujeres que tuvieron hijos en los doce meses anteriores a la fecha en que fue realizado el censo del año 2010 porque la respuesta referida a la pregunta sobre la fecha de nacimiento del último hijo nacido vivo no estaba disponible.

Cabe destacar que en Argentina existe un sistema sanitario mixto; es decir, hay un subsistema privado (el cual incluye a las obras sociales y mutuales) y uno público (que asiste a aquellas personas que no poseen cobertura de salud ${ }^{3}$ ). En este caso, se define un índice de carencia el no tener cobertura privada de salud, ya que esto podría reflejar que algún integrante del hogar tiene un trabajo formal, o bien, no posee capacidad financiera para costear un servicio de salud privado. Asimismo, se incorpora información de la población general del departamento (por ejemplo, la cobertura privada de salud y la no culminación de la escuela primaria) para conocer la realidad regional en la que están inmersas aquellas madres y sus hijos pequeños.

De igual modo, se tiene en cuenta los determinantes relacionados con la fecundidad, entendiendo que el promedio de hijos por mujer está asociado con el tamaño de los intervalos intergenésicos. Al respecto, se conoce que un intervalo menor a 24 meses aumenta el riesgo de complicaciones obstétricas y neonatales ${ }^{(13)}$.

El resultado de embarazos de niñas y adolescentes tiene peores resultados, no solo en relación a la mortalidad materna, sino también a la de los infantes comparado con el que se da en mujeres de mayor edad ${ }^{(14)}$. Siguiendo este concepto $y$, recordando que la maternidad adolescente trae implícitas ciertas desventajas desde cuestiones biológicas, pasando por un menor nivel de instrucción y otras asociadas que la colocan en una posición de vulnerabilidad es que se incluye también el promedio de hijos de madres adolescentes, debido a que tener más de un niño durante la adolescencia estaría reflejando un mayor riesgo de MI para esos niños.

Adicionalmente, se afirma que en la sociedad actual es importante poder contar con acceso a las nuevas tecnologías debido a lo significativas que se han vuelto, ya que con ellas la sociedad tiene al alcance de la mano toda la información que desee. Al respecto, Castiel y Sanz-Valero ${ }^{(15)}$ sostienen que un determinante social de la salud cuya jerarquía no es reconocida con el énfasis que amerita es el acceso a la información. El acceso a la información en salud está, en la actualidad, enormemente facilitado por las nuevas TIC, particularmente el acceso a conectividad por internet, lo cual puede ejercer gran influencia sobre la situación de salud y, en particular, sobre las inequidades en salud. Entretanto, este potencial de las TIC está amenazado si no se resuelven las inequidades de acceso a estas tecnologías, que como se ha comentado, promueven la denominada brecha digital.

Así, el uso de las TIC'S (tales como la computadora y el teléfono) son una fuente de acceso a la información que se vincula con la diferenciación social la que termina influyendo en la salud de la población. Chaparro Mendivelso ${ }^{(16)}$, manifiesta que se ha sugerido, hace más de una década y con diferentes intensidades, que la revolución digital, centrada en los computadores e internet, es un elemento importante para reconocer e identificar nuevas formas de diferenciación social y en consecuencia para delinear las nuevas distancias entre el supuesto desarrollo y el subdesarrollo; lo que algunos autores la denominan brecha digital o divisoria digital.

Para el análisis se tomó como determinantes a algunas características que reflejan el nivel de saneamiento ambiental, eliminación de excretas y hacinamiento, tenencia de agua en la cocina y métodos de cocción de los alimentos no saludables, como así también la posesión de heladera o freezer. Se hace hincapié en estas dos últimas porque están estrechamente relacionadas con la conservación y la elaboración de

construido para habitación o vivienda móvil), excluyendo casa, departamento y rancho.

C. Condiciones sanitarias: no tienen ningún tipo de retrete.

D. Asistencia escolar: tienen al menos un niño en edad escolar ( 6 a 12 años) que no asiste a la escuela.

E. Capacidad de subsistencia: tienen cuatro o más personas por miembro ocupado, cuyo jefe no haya completado el tercer grado de escolaridad primaria.

${ }^{3}$ Incluye tanto a la tenencia de una obra social (generalmente asociada a la afiliación obligatoria de los trabajadores con relación de dependencia), como a un plan de salud privado o mutual (caracterizado por la adhesión voluntaria y el pago del servicio por parte del beneficiario en su totalidad). Se excluyen los servicios de emergencias médicas. 
alimentos que son aspectos vinculados a las enfermedades diarreicas, las cuales generan una gran carga de enfermedad y muerte en los niños pequeños.

Para realizar el análisis, por un lado, y siguiendo lo que proponen Bertone ${ }^{(8)}$ y Herrero \& Bossio ${ }^{(18)}$ se toman variables censales que reflejan situaciones de carencia en algunos determinantes sociales intermediarios de la MI, las cuales demostraron una asociación con la MI a nivel departamental en Argentina. Entre estas variables se consideran ciertas características de las personas, de los hogares y también de las viviendas, teniendo en cuenta que se refieren a los determinantes de los ámbitos familiares, residenciales, y regionales -tal como se propone desde el Modelo de Evaluación Global del Ambiente ${ }^{(11)}$ - ya que en conjunto involucran a varias esferas de dicho modelo. A esta clase de modelos se los denomina Determinantes Sociales Intermediarios de la Mortalidad Infantil, de acuerdo al marco conceptual de la CDSS mencionado en el marco teórico ${ }^{(10)}$.

A continuación, en la Tabla 1 se presentan las variables y categorías que serán tomadas como determinantes en este artículo:

Tabla 1. Determinantes Sociales Intermediarios de la Mortalidad Infantil

\begin{tabular}{|c|c|c|c|}
\hline & Determinantes & Variable Censal & $\begin{array}{l}\text { Categoría de } \\
\text { inclusión }\end{array}$ \\
\hline \multirow{3}{*}{ 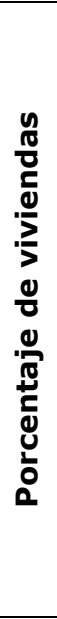 } & $\begin{array}{l}\text { Vivienda con } \\
\text { materiales } \\
\text { deficientes }\end{array}$ & $\begin{array}{l}\text { Refiere a la calidad de los materiales con que } \\
\text { están construidas las viviendas (material } \\
\text { predominante de los pisos y techos), } \\
\text { teniendo en cuenta la solidez, resistencia y } \\
\text { capacidad de aislamiento, así como también } \\
\text { su terminación. }\end{array}$ & $\begin{array}{l}\text { La vivienda presenta } \\
\text { materiales de baja } \\
\text { calidad en pisos y } \\
\text { techos. }\end{array}$ \\
\hline & $\begin{array}{l}\text { Saneamiento } \\
\text { ambiental } \\
\text { deficiente }\end{array}$ & $\begin{array}{l}\text { Refiere al tipo de instalaciones con el que } \\
\text { cuentan las viviendas para su saneamiento. } \\
\text { Para este indicador, se utilizan las variables } \\
\text { procedencia del agua y tipo de desagüe. }\end{array}$ & $\begin{array}{l}\text { Saneamiento } \\
\text { Insuficiente. }\end{array}$ \\
\hline & $\begin{array}{l}\text { De zona rural } \\
\text { dispersa }\end{array}$ & $\begin{array}{l}\text { Diferencia los espacios según constituyan } \\
\text { agrupamientos en localidades y el tamaño de } \\
\text { dichas localidades. Se procura identificar a } \\
\text { las viviendas que por su ubicación es más } \\
\text { difícil que tengan acceso a servicios de salud. }\end{array}$ & $\begin{array}{l}\text { Rural dispersa: } \\
\text { viviendas que no se } \\
\text { encuentren en } \\
\text { manzanas divididas por } \\
\text { calles, constituyen la } \\
\text { llamada población rural } \\
\text { dispersa. }\end{array}$ \\
\hline \multirow{5}{*}{ 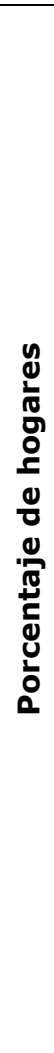 } & $\begin{array}{l}\text { Condiciones de la } \\
\text { cocina deficitarias }\end{array}$ & Instalación de agua en la cocina. & $\begin{array}{l}\text { Sin instalación de agua } \\
\text { o sin cocina. }\end{array}$ \\
\hline & $\begin{array}{l}\text { Sin heladera o } \\
\text { freezer }\end{array}$ & $\begin{array}{l}\text { Refiere a la disponibilidad de un } \\
\text { artefacto/aparato doméstico consistente en } \\
\text { un receptáculo con paredes aislantes provisto } \\
\text { de un motor que genera bajas temperaturas } \\
\text { que permiten mantener frescos los alimentos } \\
\text { y bebidas en su interior. }\end{array}$ & $\begin{array}{l}\text { No tiene heladera ni } \\
\text { freezer. }\end{array}$ \\
\hline & $\begin{array}{l}\text { Métodos de cocción } \\
\text { poco saludables }\end{array}$ & $\begin{array}{l}\text { Refiere a la sustancia (que tiene el atributo } \\
\text { de arder) o fuente de energía que se utiliza } \\
\text { con mayor frecuencia para la cocción de los } \\
\text { alimentos, tales como: combustibles gaseo- } \\
\text { sos (gas natural o licuado), sólidos (carbón, } \\
\text { productos del carbón, leña), petróleo y } \\
\text { derivados (kerosene) y otros como la } \\
\text { electricidad y el alcohol. }\end{array}$ & $\begin{array}{l}\text { Leña o carbón. La } \\
\text { utilización de medios } \\
\text { de combustión es } \\
\text { utilizada como un } \\
\text { proxy de servicios y } \\
\text { salubridad para la } \\
\text { preparación de los } \\
\text { alimentos. }\end{array}$ \\
\hline & Con hacinamiento & $\begin{array}{l}\text { Representa el cociente entre la cantidad total } \\
\text { de personas del hogar y la cantidad total de } \\
\text { habitaciones o piezas de que dispone el } \\
\text { mismo (sin contar baño/s y cocina/s). }\end{array}$ & $\begin{array}{l}\text { Más de } 3 \text { personas por } \\
\text { cuarto. }\end{array}$ \\
\hline & Sin retrete & $\begin{array}{l}\text { Refiere a la disponibilidad de un espacio } \\
\text { cerrado por paredes que se elevan del suelo } \\
\text { hasta el techo, o por tabiques (pared que se } \\
\text { eleva desde el suelo hasta una altura de dos } \\
\text { metros), utilizado por el hogar para la } \\
\text { evacuación de excretas. El baño/letrina } \\
\text { puede estar dentro o fuera de la vivienda y } \\
\text { ser de uso exclusivo del hogar o compartido } \\
\text { con otros hogares. }\end{array}$ & No tiene. \\
\hline
\end{tabular}




\begin{tabular}{|c|c|c|c|}
\hline & $\begin{array}{l}\text { Con NBI de } \\
\text { subsistencia }\end{array}$ & $\begin{array}{l}\text { Las Necesidades Básicas Insatisfechas (NBI) } \\
\text { fueron definidas según la metodología } \\
\text { utilizada en La pobreza en la Argentina (serie } \\
\text { Estudios INDEC, No } 1 \text {, Buenos Aires, 1984). } \\
\text { Se toma solo un aspecto de este indicador: } \\
\text { Escolaridad }\end{array}$ & $\begin{array}{l}\text { Tienen cuatro o más } \\
\text { personas por miembro } \\
\text { ocupado, cuyo jefe no } \\
\text { haya completado el tercer } \\
\text { grado de escolaridad } \\
\text { primaria. }\end{array}$ \\
\hline & Sin electricidad & Tenencia de electricidad & No tiene. \\
\hline & Sin computadora & $\begin{array}{l}\text { Indica si el hogar cuenta con un aparato } \\
\text { electrónico que se utiliza para el almacenaje, } \\
\text { procesamiento de información (datos, } \\
\text { palabras, imágenes, etc). }\end{array}$ & $\begin{array}{l}\text { No accede al uso de } \\
\text { TICS. Esto es reconocido } \\
\text { como una fuente de } \\
\text { acceso a la información. }\end{array}$ \\
\hline & $\begin{array}{l}\text { Sin teléfono } \\
\text { celular }\end{array}$ & $\begin{array}{l}\text { Teléfono móvil desde el que se pueden } \\
\text { efectuar y recibir llamadas, en una red } \\
\text { telefónica por células. Se considera su } \\
\text { disponibilidad en el hogar si al menos uno de } \\
\text { sus integrantes tiene un teléfono celular. }\end{array}$ & $\begin{array}{l}\text { No posee Celular. Al igual } \\
\text { que con la computadora } \\
\text { esta tecnología permite } \\
\text { acceder a información de } \\
\text { manera más accesible } \\
\text { que la PC. }\end{array}$ \\
\hline \multirow{2}{*}{ 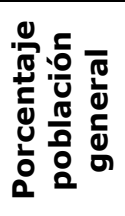 } & $\begin{array}{l}\text { Sin cobertura de } \\
\text { salud }\end{array}$ & $\begin{array}{l}\text { Refiere a la cobertura de salud, ya sea obra } \\
\text { social (incluye PAMI), prepaga o plan estatal } \\
\text { de salud. }\end{array}$ & $\begin{array}{l}\text { No tiene obra social, } \\
\text { prepaga o plan estatal. }\end{array}$ \\
\hline & $\begin{array}{l}\text { Bajo nivel de } \\
\text { instrucción }\end{array}$ & $\begin{array}{l}\text { Máximo nivel de instrucción de población } \\
\text { general mayor de } 14 \text { años. }\end{array}$ & Primaria incompleta. \\
\hline \multirow{2}{*}{ 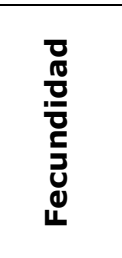 } & $\begin{array}{l}\text { Promedio de hijos } \\
\text { por mujer en edad } \\
\text { fértil }\end{array}$ & Mujeres de entre 14 y 49 años de edad. & Número de hijos. \\
\hline & $\begin{array}{l}\text { Promedio de hijos } \\
\text { por mujer menor } \\
\text { de } 20 \text { años de } \\
\text { edad }\end{array}$ & Mujeres menores de 20 años de edad. & Número de hijos. \\
\hline
\end{tabular}

Fuente: Elaboración propia en base a datos extraídos del Censo de población, hogares y viviendas 2010 (base ampliada).

Por otro lado, se realiza el análisis espacial de la mortalidad infantil tanto neonatal como pos-neonatal de causas reducibles. Se habla de MI neonatal cuando la muerte del niño ocurre entre el momento del nacimiento y el vigésimo séptimo día, mientras que la pos-neonatal es la que sucede entre el día 28 hasta el día 364 de vida. Entonces, las defunciones de los niños se agrupan de acuerdo al momento de su muerte y, además, se ordenan por departamento de residencia de la madre y año de ocurrencia, tal como se agrupan los nacimientos.

Las causas de muerte infantiles se clasificaron de acuerdo a las recomendaciones oficiales realizadas para los años estudiados ${ }^{(19)}$, y se tomaron en conjunto todas las causas consideradas reducibles neonatales y posneonatales respectivamente.

El análisis espacial se realiza mediante el software libre SaTScan ${ }^{4}$. Este programa se basa en el supuesto de que un evento epidemiológico de interés posee una distribución de tipo Poisson, que es la distribución probabilística utilizada para analizar variables discretas que representan eventos $\operatorname{raros}^{(20)}$. A su vez, permite analizar el riesgo de que ocurra dicho evento dentro de 'espacios' o 'ventanas', y se hace el análisis (para unidad geográfica) a partir de los casos observados, la población en riesgo, y de los casos esperados. Es decir, las defunciones infantiles constituyen los casos observados, la población en riesgo corresponde a los nacimientos y las unidades geográficas a los departamentos de residencia de las madres de los niños, los que constituyen, como unidad o agrupados, las ventanas o espacios mencionados.

La hipótesis nula que se pone a prueba es si el riesgo de ocurrencia del evento se mantiene constante a lo largo del espacio. Si se rechaza la hipótesis nula, se encuentra evidencia que existe un conglomerado de $\operatorname{casos}^{(21)}$. Por su parte, la

\footnotetext{
${ }^{4}$ Software: Kulldorff M. and Information Management Services, Inc. SaTScanTM v8.0: Software for the spatial and spacetime scan statistics. http://www.SaTScan.org/ , 2009.
} 
hipótesis alternativa es que para al menos una de las "ventanas" el riesgo es diferente dentro y fuera de él, es decir que, ese espacio conforma un conglomerado.

Algunas limitaciones de este trabajo se relacionan con la calidad de los datos de las fuentes seleccionadas. Al respecto, según estudios realizados por Fernández, Guevel, Krupitzki, Marconi y Massa(22) y el Ministerio de Salud ${ }^{(23)}$ acerca de los errores de las estadísticas vitales, mencionan que existen evidencias de una omisión de nacimientos a nivel país del 6\%, a partir de estimaciones indirectas tanto con datos del censo 2001 como el del 2010. Además indican que existe una omisión diferencial con el interior del país, sobresaliendo Santiago del Estero como la jurisdicción con mayor omisión del NOA. Asimismo, en ellos se aplicaron técnicas directas de evaluación, indicando las omisiones de nacimientos que se hicieron en algunos de los principales centros asistenciales de las provincias.

De esto, surgen valores de omisión de $7 \%$ en Salta, $10 \%$ en la Rioja, $15 \%$ en Tucumán y $20 \%$ en Santiago del Estero. En dichas técnicas directas también se evaluó la cobertura de las defunciones infantiles en centros asistenciales seleccionados, resultando sin omisión en Salta, la omisión en La Rioja fue de $9 \%$, entre $9 \%$ y en $15 \%$ en los hospitales de Tucumán, y en $78 \%$ y $46 \%$ en los centros asistenciales de Santiago del Estero. Estos valores no reflejan necesariamente la omisión de toda la provincia ya que surgen de muestras intencionales, sin embargo permiten una aproximación de la completitud de las estadísticas vitales en la región, lo que en Santiago del Estero y Tucumán podría implicar una subestimación de la mortalidad infantil reducible.

Otro aspecto que hay que tener en cuenta en este estudio es que se trata de un estudio ecológico, de modo que lo hallado en relación a la población residente en un departamento no necesariamente refleja o significa que sea así para todos y cada uno de los individuos que habitan ese territorio, sino para el conjunto. Es decir, al interior del departamento o del conjunto de departamentos los individuos pueden tener un riesgo distinto al hallado en el conjunto.

\section{RESULTADOS}

En el NOA, existen 36 departamentos que tienen más del 50\% de viviendas deficientes. Algunos hogares no cuentan con cocina o agua en la cocina. Entre los principales resultados que se presentan en la tabla 2, puede señalarse que hay algunos departamentos en los que el $50 \%$ de los hogares no tienen computadora y la población sin obra social ronda entre el 15 y $79 \%$.

Tabla 2. Determinantes de la Salud Infantil en los departamentos del NOA (20102014).

\begin{tabular}{|c|c|c|c|c|c|c|}
\hline Entidad & Determinante & $\begin{array}{l}\mathbf{D} \\
\mathbf{N}\end{array}$ & Mínimo & $\begin{array}{l}\text { tadísticos } \\
\text { Máximo }\end{array}$ & $\begin{array}{l}\text { descrip } \\
\text { Media }\end{array}$ & $\begin{array}{l}\text { ptivos } \\
\text { Desviación } \\
\text { estándar }\end{array}$ \\
\hline \multirow{3}{*}{$\begin{array}{l}\text { Porcentaje } \\
\text { de viviendas }\end{array}$} & Vivienda deficiente & 117 & $2 \%$ & $97 \%$ & $28 \%$ & 0,208 \\
\hline & $\begin{array}{l}\text { Saneamiento ambiental } \\
\text { deficiente }\end{array}$ & 117 & $10 \%$ & $90 \%$ & $34 \%$ & 0,165 \\
\hline & Rural dispersa & 117 & $0 \%$ & $76 \%$ & $27 \%$ & 0,202 \\
\hline \multirow{8}{*}{$\begin{array}{l}\text { Porcentaje } \\
\text { de hogares }\end{array}$} & $\begin{array}{l}\text { Combustible para } \\
\text { cocinar poco saludable }\end{array}$ & 117 & $1 \%$ & $77 \%$ & $22 \%$ & 0,184 \\
\hline & Sin inodoro o letrina & 117 & $2 \%$ & $30 \%$ & $10 \%$ & 0,055 \\
\hline & $\begin{array}{l}\text { Sin cocina o instalación } \\
\text { de agua en la cocina }\end{array}$ & 117 & $12 \%$ & $95 \%$ & $51 \%$ & 0,199 \\
\hline & Sin electricidad & 117 & $0 \%$ & $47 \%$ & $10 \%$ & 0,101 \\
\hline & Sin computadora & 117 & $50 \%$ & $96 \%$ & $78 \%$ & 0,106 \\
\hline & Sin teléfono celular & 117 & $10 \%$ & $88 \%$ & $25 \%$ & 0,150 \\
\hline & Sin heladera & 117 & $4 \%$ & $84 \%$ & $24 \%$ & 0,179 \\
\hline & Con NBI de Subsistencia & 117 & $0 \%$ & $5 \%$ & $2 \%$ & 0,010 \\
\hline Población general & $\begin{array}{l}\text { Población sin Obra } \\
\text { Social }\end{array}$ & 117 & $15 \%$ & $79 \%$ & $50 \%$ & 0,126 \\
\hline
\end{tabular}




\begin{tabular}{llccccc}
\hline & $\begin{array}{l}\text { Primaria incompleta } \\
\begin{array}{l}\text { Número de hijos } \\
\text { nacidos vivos de }\end{array}\end{array}$ & 117 & $8 \%$ & $46 \%$ & $22 \%$ & 0,090 \\
$\begin{array}{l}\text { mujeres de edad fértil } \\
\text { Número de hijos } \\
\text { nacidos vivos de }\end{array}$ & 117 & 1,1 & 1,7 & 1,2 & 0,081 \\
\hline Fecundidad & adoscentes & 3,9 & 0,307 \\
\hline
\end{tabular}

Nota: D: departamentos.

Fuente: elaboración propia en base a datos del Censo Nacional de población, hogares y viviendas. 2010, INDEC.

Por otra parte, según el análisis espacial de la mortalidad efectuado, se detectaron dos aglomerados significativos de departamentos de alto riesgo para la mortalidad infantil por causas reducibles (ver Figura 1 ).

La zona de mayor riesgo de mortalidad neonatal está conformada por algunos departamentos de las provincias de Salta y Jujuy que se enumeran en la tabla 3. Los niños que murieron antes de su 28 día de vida, cuyas madres residían en este conjunto de departamentos, presentaron un $76 \%$ más de riesgo de morir que aquellos cuyas madres residían en el resto del NOA. De ese conjunto, los departamentos jujeños de Cochinoca y Rinconada presentaron el mayor riesgo, mientras Santa Victoria es el de mayor riesgo en Salta.

El otro conjunto de departamentos que resultó significativo y con alto riesgo para la mortalidad infantil pos-neonatal está formado sólo por departamentos de la provincia de Santiago del Estero (Tabla 4). Los niños, cuyas madres residían en esos departamentos santiagueños, tuvieron un $40 \%$ más de riesgo de morir por causas reducibles que el resto de la región. En particular, Salavina y Ojo de Agua son los departamentos de mayor riesgo dentro del aglomerado.

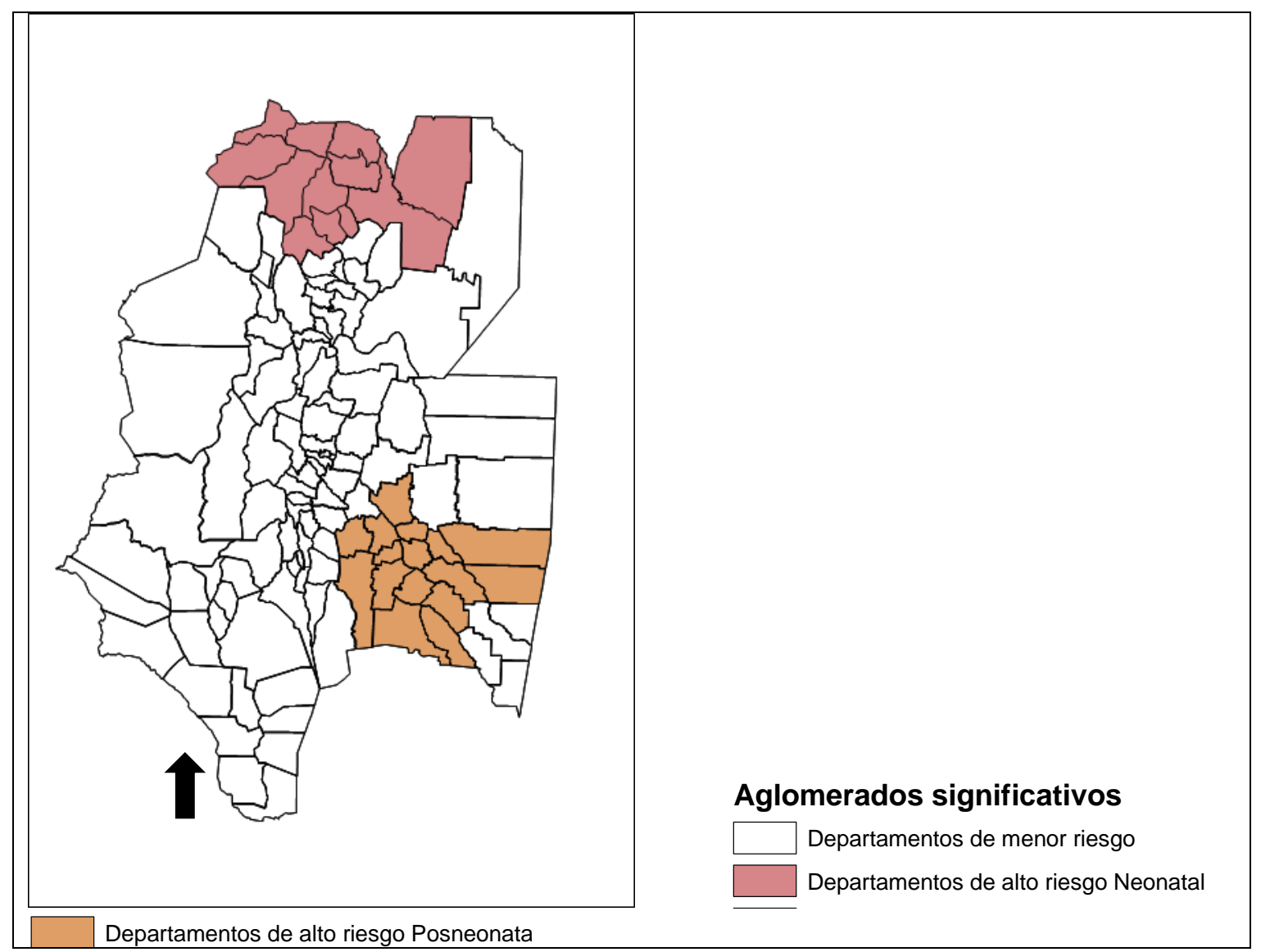

Fuente: elaboración propia en base a datos provistos por la Dirección de Estadísticas e Información de Salud del Ministerio de Salud de la Nación Argentina (2010-2014).

Figura 1. Aglomerado de departamento de alto riesgo de mortalidad infantil neonatal y pos-neonatal por causas reducibles. NOA (2010-2014) 
Cuando se identificaron los departamentos de alto riesgo para la MI, se procedió a realizar una prueba de comparación de medias de muestras independientes. Es decir, las medias de los determinantes sociales de la salud mencionados en la primera parte divididos en dos grupos: uno constituido por aquellos departamentos que conforman conglomerados de alto riesgo y el otro por los que no se incluyeron en ellos. En la Tabla 3 se observan las comparaciones de las medias de cada determinante seleccionado en este estudio y en la Tabla 4 la prueba $T$ para muestras independientes realizada a cada uno de ellos. Los resultados obtenidos mediante dicho test permiten concluir que existen algunos determinantes donde hay diferencias estadísticamente significativas en sus medias entre los departamentos de alto riesgo de mortalidad neonatal y el resto de la región, utilizando un nivel de confianza del $95 \%$.

Los departamentos de alto riesgo para la mortalidad infantil neonatal presentan peores condiciones de carencia en relación a un conjunto de DSS como saneamiento ambiental, el hecho de vivir en una zona rural dispersa o no tener inodoro o letrina.

Tabla 3. Estadísticos descriptivos: los determinantes sociales en departamentos del NOA según su riesgo para la mortalidad infantil por causas reducibles neonatales.

\begin{tabular}{|c|c|c|c|c|c|c|}
\hline Entidad & Determinante & Riesgo & $\mathbf{N}$ & Media & $\begin{array}{l}\text { Desviación } \\
\text { estándar }\end{array}$ & $\begin{array}{l}\text { Media } \\
\text { de error } \\
\text { estándar }\end{array}$ \\
\hline \multirow{6}{*}{ Porcentaje de viviendas } & \multirow{2}{*}{ Vivienda deficiente } & Menor riesgo & 101 & 0,268 & 0,215 & 0,021 \\
\hline & & Mayor riesgo & 16 & 0,339 & 0,150 & 0,038 \\
\hline & \multirow{2}{*}{$\begin{array}{l}\text { Saneamiento ambiental } \\
\text { deficiente }\end{array}$} & Menor riesgo & 101 & 0,323 & 0,160 & 0,016 \\
\hline & & Mayor riesgo & 16 & 0,457 & 0,146 & 0,037 \\
\hline & \multirow{2}{*}{ Rural dispersa } & Menor riesgo & 101 & 0,256 & 0,203 & 0,020 \\
\hline & & Mayor riesgo & 16 & 0,393 & 0,158 & 0,039 \\
\hline \multirow{16}{*}{ Porcentaje de hogares } & \multirow{2}{*}{$\begin{array}{l}\text { Combustible para cocinar } \\
\text { poco saludable }\end{array}$} & Menor riesgo & 101 & 0,202 & 0,183 & 0,018 \\
\hline & & Mayor riesgo & 16 & 0,344 & 0,145 & 0,036 \\
\hline & \multirow{2}{*}{ Sin inodoro o letrina } & Menor riesgo & 101 & 0,092 & 0,054 & 0,005 \\
\hline & & Mayor riesgo & 16 & 0,136 & 0,049 & 0,012 \\
\hline & \multirow{2}{*}{$\begin{array}{l}\text { Sin espacio cocina o } \\
\text { instalación de agua en la } \\
\text { cocina }\end{array}$} & Menor riesgo & 101 & 0,480 & 0,191 & 0,019 \\
\hline & & Mayor riesgo & 16 & 0,677 & 0,168 & 0,042 \\
\hline & \multirow{2}{*}{ Sin electricidad } & Menor riesgo & 101 & 0,087 & 0,099 & 0,010 \\
\hline & & Mayor riesgo & 16 & 0,166 & 0,087 & 0,022 \\
\hline & \multirow{2}{*}{ Sin computadora } & Menor riesgo & 101 & 0,770 & 0,105 & 0,010 \\
\hline & & Mayor riesgo & 16 & 0,867 & 0,073 & 0,018 \\
\hline & \multirow{2}{*}{ Sin teléfono celular } & Menor riesgo & 101 & 0,256 & 0,159 & 0,016 \\
\hline & & Mayor riesgo & 16 & 0,181 & 0,027 & 0,007 \\
\hline & \multirow{2}{*}{ Sin heladera } & Menor riesgo & 101 & 0,237 & 0,188 & 0,019 \\
\hline & & Mayor riesgo & 16 & 0,282 & 0,104 & 0,026 \\
\hline & \multirow{2}{*}{ Con NBI de subsistencia } & Menor riesgo & 101 & 0,018 & 0,010 & 0,001 \\
\hline & & Mayor riesgo & 16 & 0,023 & 0,010 & 0,003 \\
\hline \multirow{4}{*}{ Población general } & \multirow{2}{*}{ Población sin obra social } & Menor riesgo & 101 & 0,479 & 0,116 & 0,012 \\
\hline & & Mayor riesgo & 16 & 0,652 & 0,077 & 0,019 \\
\hline & \multirow{2}{*}{ Primaria incompleta } & Menor riesgo & 101 & 0,211 & 0,086 & 0,009 \\
\hline & & Mayor riesgo & 16 & 0,309 & 0,072 & 0,018 \\
\hline \multirow{4}{*}{ Fecundidad } & \multirow{2}{*}{$\begin{array}{l}\text { Número de hijos nacidos } \\
\text { vivos de mujeres de edad } \\
\text { fértil }\end{array}$} & Menor riesgo & 101 & 2,998 & 0,309 & 0,031 \\
\hline & & Mayor riesgo & 16 & 3,185 & 0,246 & 0,061 \\
\hline & \multirow{2}{*}{$\begin{array}{l}\text { Número de hijos nacidos } \\
\text { vivos de adolescentes }\end{array}$} & Menor riesgo & 101 & 1,232 & 0,086 & 0,009 \\
\hline & & Mayor riesgo & 16 & 1,229 & 0,044 & 0,011 \\
\hline
\end{tabular}

Fuente: elaboración propia en base a datos del Censo Nacional de población, hogares y viviendas. 2010, INDEC. 
Otros aspectos donde se encontraron situaciones más deficientes fueron en la cocina: no tener un espacio para cocinar o agua instalada en el mismo y cocinar con combustibles poco saludables, son una de las situaciones más precarias en estos departamentos caracterizados por poseer mayor MI neonatal reducible.

También presentaron peores condiciones, como no tener electricidad o computadora. De igual manera, se encontraron diferencias en aspectos que hacen más vulnerables a la población general como su nivel de instrucción o cobertura de salud. El número de hijos nacidos vivos de las mujeres en edad fértil también fue disímil entre estos dos grupos, con un mayor número promedio en el grupo de riesgo.

En el grupo de departamentos de alto riesgo, para la mortalidad pos-neonatal (departamentos de la provincia de Santiago del Estero) se destacó que había mayor proporción de viviendas con materiales de baja calidad, sin acceso a electricidad, coincidente con menor porcentaje de hogares con computadora.

Tabla 4. Prueba de muestras independientes de determinantes sociales de la mortalidad infantil neonatal en departamentos de alto riesgo y el resto de los departamentos del NOA (2010-2014)

\begin{tabular}{|c|c|c|c|c|c|c|c|}
\hline \multirow[b]{3}{*}{ Entidad } & \multirow[b]{3}{*}{ Determinantes } & & \multicolumn{5}{|c|}{ Prueba T para la igualdad de medias } \\
\hline & & & \multirow[b]{2}{*}{$\mathbf{T}$} & \multirow{2}{*}{$\begin{array}{l}\text { Sig. } \\
\text { (bilateral) }\end{array}$} & \multirow[b]{2}{*}{ DM } & \multicolumn{2}{|c|}{ IC } \\
\hline & & & & & & $\mathbf{I}$ & $\mathbf{S}$ \\
\hline \multirow{3}{*}{$\begin{array}{l}\text { Porcentaje } \\
\text { de } \\
\text { viviendas }\end{array}$} & Vivienda deficiente & VI & $-1,283$ & 0,202 & $-0,072$ & $-0,182$ & 0,039 \\
\hline & $\begin{array}{l}\text { Saneamiento ambiental } \\
\text { deficiente }\end{array}$ & VI & $-3,132$ & 0,002 & $-0,134$ & $-0,218$ & $-0,049$ \\
\hline & Rural dispersa & VI & $-2,583$ & 0,011 & $-0,137$ & $-0,242$ & $-0,032$ \\
\hline \multirow{8}{*}{$\begin{array}{l}\text { Porcentaje } \\
\text { de hogares }\end{array}$} & $\begin{array}{l}\text { Combustible para cocinar } \\
\text { poco saludable }\end{array}$ & VI & $-2,966$ & 0,004 & $-0,142$ & $-0,237$ & $-0,047$ \\
\hline & Sin inodoro o letrina & VI & $-3,073$ & 0,003 & $-0,044$ & $-0,073$ & $-0,016$ \\
\hline & $\begin{array}{l}\text { Sin espacio cocina o } \\
\text { Instalación de agua en la } \\
\text { cocina }\end{array}$ & VI & $-3,888$ & 0,000 & $-0,197$ & $-0,297$ & $-0,097$ \\
\hline & Sin electricidad & VI & $-3,030$ & 0,003 & $-0,079$ & $-0,131$ & $-0,027$ \\
\hline & Sin computadora & NVI & $-4,590$ & 0,000 & $-0,097$ & $-0,140$ & $-0,053$ \\
\hline & Sin teléfono celular & NVI & 4,379 & 0,000 & 0,075 & 0,041 & 0,109 \\
\hline & Sin heladera & VI & $-0,922$ & 0,358 & $-0,044$ & $-0,140$ & 0,051 \\
\hline & Con NBI de Subsistencia & VI & $-1,746$ & 0,084 & $-0,005$ & $-0,010$ & 0,001 \\
\hline \multirow{2}{*}{$\begin{array}{l}\text { Población } \\
\text { general }\end{array}$} & Población sin Obra Social & NVI & $-7,721$ & 0,000 & $-0,173$ & $-0,219$ & $-0,127$ \\
\hline & $\begin{array}{l}\text { Población general } \\
\text { primaria incompleta }\end{array}$ & VI & $-4,353$ & 0,000 & $-0,098$ & $-0,143$ & $-0,054$ \\
\hline \multirow{2}{*}{ Fecundidad } & $\begin{array}{l}\text { Número de hijos nacidos } \\
\text { vivos de mujeres de edad } \\
\text { fértil }\end{array}$ & VI & $-2,307$ & 0,023 & $-0,187$ & $-0,348$ & $-0,027$ \\
\hline & $\begin{array}{l}\text { Número de hijos nacidos } \\
\text { vivos de mujeres } \\
\text { adolescentes }\end{array}$ & VI & 0,148 & 0,882 & 0,003 & $-0,040$ & 0,047 \\
\hline
\end{tabular}


Tabla 5. Estadísticos descriptivos de los determinantes sociales en departamentos del NOA según su riesgo para la Mortalidad Infantil por causas reducibles pos-neonatales (2010-2014)

\begin{tabular}{|c|c|c|c|c|c|c|}
\hline Entidad & DSS & Riesgo & $\mathbf{N}$ & Media & DS & $\begin{array}{l}\text { Media de } \\
\text { error } \\
\text { estándar }\end{array}$ \\
\hline \multirow{6}{*}{ Porcentaje de viviendas } & \multirow{2}{*}{ Vivienda deficiente } & Menor riesgo & 105 & 0,254 & 0,199 & 0,019 \\
\hline & & Mayor riesgo & 12 & 0,485 & 0,178 & 0,051 \\
\hline & \multirow{2}{*}{$\begin{array}{l}\text { Saneamiento ambiental } \\
\text { deficiente }\end{array}$} & Menor riesgo & 105 & 0,336 & 0,168 & 0,016 \\
\hline & & Mayor riesgo & 12 & 0,384 & 0,131 & 0,038 \\
\hline & \multirow{2}{*}{ Rural dispersa } & Menor riesgo & 105 & 0,269 & 0,204 & 0,020 \\
\hline & & Mayor riesgo & 12 & 0,323 & 0,182 & 0,053 \\
\hline \multirow{16}{*}{ Porcentaje de hogares } & \multirow{2}{*}{$\begin{array}{l}\text { Combustible para cocinar } \\
\text { poco saludable }\end{array}$} & Menor riesgo & 105 & 0,196 & 0,166 & 0,016 \\
\hline & & Mayor riesgo & 12 & 0,439 & 0,194 & 0,056 \\
\hline & \multirow{2}{*}{ Sin inodoro o letrina } & Menor riesgo & 105 & 0,095 & 0,056 & 0,006 \\
\hline & & Mayor riesgo & 12 & 0,121 & 0,038 & 0,011 \\
\hline & \multirow{2}{*}{$\begin{array}{l}\text { Sin cocina o Instalación } \\
\text { de agua en la cocina }\end{array}$} & Menor riesgo & 105 & 0,486 & 0,196 & 0,019 \\
\hline & & Mayor riesgo & 12 & 0,689 & 0,127 & 0,037 \\
\hline & \multirow{2}{*}{ Sin electricidad } & Menor riesgo & 105 & 0,088 & 0,094 & 0,009 \\
\hline & & Mayor riesgo & 12 & 0,184 & 0,117 & 0,034 \\
\hline & \multirow{2}{*}{ Sin computadora } & Menor riesgo & 105 & 0,776 & 0,107 & 0,010 \\
\hline & & Mayor riesgo & 12 & 0,848 & 0,079 & 0,023 \\
\hline & \multirow{2}{*}{ Sin teléfono celular } & Menor riesgo & 105 & 0,214 & 0,098 & 0,010 \\
\hline & & Mayor riesgo & 12 & 0,519 & 0,236 & 0,068 \\
\hline & \multirow{2}{*}{ Sin heladera } & Menor riesgo & 105 & 0,210 & 0,143 & 0,014 \\
\hline & & Mayor riesgo & 12 & 0,531 & 0,210 & 0,061 \\
\hline & \multirow{2}{*}{ Con NBI de Subsistencia } & Menor riesgo & 105 & 0,019 & 0,010 & 0,001 \\
\hline & & Mayor riesgo & 12 & 0,023 & 0,009 & 0,003 \\
\hline \multirow{4}{*}{ Población general } & \multirow{2}{*}{ Población sin Obra Social } & Menor riesgo & 105 & 0,492 & 0,127 & 0,012 \\
\hline & & Mayor riesgo & 12 & 0,591 & 0,074 & 0,021 \\
\hline & \multirow{2}{*}{ Primaria incompleta } & Menor riesgo & 105 & 0,219 & 0,090 & 0,009 \\
\hline & & Mayor riesgo & 12 & 0,272 & 0,077 & 0,022 \\
\hline \multirow{4}{*}{ Fecundidad } & \multirow{2}{*}{$\begin{array}{l}\text { Número de hijos nacidos } \\
\text { vivos de mujeres de edad } \\
\text { fértil }\end{array}$} & Menor riesgo & 105 & 2,994 & 0,282 & 0,028 \\
\hline & & Mayor riesgo & 12 & 3,281 & 0,399 & 0,115 \\
\hline & \multirow{2}{*}{$\begin{array}{l}\text { Número de hijos nacidos } \\
\text { vivos de adolescentes }\end{array}$} & Menor riesgo & 105 & 1,228 & 0,077 & 0,008 \\
\hline & & Mayor riesgo & 12 & 1,266 & 0,108 &, 031 \\
\hline
\end{tabular}

Fuente: elaboración propia en base a datos del Censo Nacional de población, hogares y viviendas. 2010, INDEC.

En la siguiente Tabla se exhibe que estos departamentos presentan mayor proporción de hogares sin cocina y que cocinan con combustibles poco saludables. Esta subpoblación cuenta con bajo nivel de instrucción y el porcentaje de población sin cobertura de salud es mayor que la del resto del NOA, así como también presentan un mayor número promedio de hijos nacidos vivos. 
Tabla 6. Prueba $T$ de muestras independientes de determinantes sociales de la Mortalidad Infantil pos-neonatal en departamentos de alto riesgo y el resto de los departamentos del NOA (2010-2014)

\begin{tabular}{|c|c|c|c|c|c|c|c|}
\hline \multirow{3}{*}{ Entidad } & \multirow{3}{*}{ Determinantes } & & \multicolumn{5}{|c|}{ Prueba $\mathbf{T}$ para la igualdad de medias } \\
\hline & & & \multirow{2}{*}{$\mathbf{T}$} & \multirow{2}{*}{$\begin{array}{l}\text { Sig. } \\
\text { (bilat } \\
\text { eral) }\end{array}$} & \multirow{2}{*}{ DM } & \multicolumn{2}{|c|}{ IC } \\
\hline & & & & & & $\mathbf{I}$ & $\mathbf{S}$ \\
\hline \multirow{3}{*}{$\begin{array}{l}\text { Porcentaje de } \\
\text { viviendas }\end{array}$} & Vivienda deficiente & VI & $-3,858$ & 0,000 & $-0,231$ & $-0,350$ & $-0,113$ \\
\hline & $\begin{array}{l}\text { Saneamiento ambiental } \\
\text { deficiente }\end{array}$ & VI &,- 952 & 0,343 & $-0,048$ & $-0,147$ & 0,052 \\
\hline & Rural dispersa & VI &,- 877 & 0,382 & $-0,054$ & $-0,176$ & 0,068 \\
\hline \multirow{8}{*}{$\begin{array}{l}\text { Porcentaje de } \\
\text { hogares }\end{array}$} & $\begin{array}{l}\text { Combustible para cocinar } \\
\text { poco saludable }\end{array}$ & VI & $-4,718$ & 0,000 & $-0,243$ & $-0,345$ & $-0,141$ \\
\hline & Sin inodoro o letrina & VI & $-1,554$ & 0,123 & $-0,026$ & $-0,059$ & 0,007 \\
\hline & $\begin{array}{l}\text { Sin cocina o Instalación de } \\
\text { agua en la cocina }\end{array}$ & VI & $-3,511$ & 0,001 & $-0,203$ & $-0,318$ & $-0,089$ \\
\hline & Sin electricidad & VI & $-3,266$ & 0,001 & $-0,096$ & $-0,155$ & $-0,038$ \\
\hline & Sin computadora & VI & $-2,270$ & 0,025 & $-0,072$ & $-0,135$ & 0,009 \\
\hline & Sin teléfono celular & NVI & $-4,427$ & 0,001 & $-0,305$ & $-0,455$ & $-0,154$ \\
\hline & Sin heladera & NVI & $-5,148$ & 0,000 & $-0,321$ & $-0,457$ & $-0,185$ \\
\hline & Con NBI de Subsistencia & NVI & $-1,566$ & 0,138 & $-0,004$ & $-0,010$ & 0,002 \\
\hline \multirow{2}{*}{ Población general } & Población sin Obra Social & NVI & $-3,979$ & 0,001 & $-0,098$ & $-0,150$ & $-0,047$ \\
\hline & $\begin{array}{l}\text { Población general primaria } \\
\text { incompleta }\end{array}$ & VI & $-1,952$ & 0,053 & $-0,053$ & $-0,107$ & 0,001 \\
\hline \multirow{2}{*}{ Fecundidad } & $\begin{array}{l}\text { Número de hijos nacidos } \\
\text { vivos de mujeres de edad } \\
\text { fértil }\end{array}$ & NVI & $-2,424$ & 0,032 & $-0,287$ & $-0,545$ & $-0,030$ \\
\hline & $\begin{array}{l}\text { Número de hijos nacidos } \\
\text { vivos de mujeres } \\
\text { adolescentes }\end{array}$ & VI & $-1,559$ & 0,122 & $-0,038$ & $-0,087$ & 0,010 \\
\hline
\end{tabular}

Nota: IC: $95 \%$ de intervalo de confianza de la diferencia; I: límite inferior; S: límite superior; DM: Diferencia de medias; VI: se asumen varianzas iguales; NVI: No se asumen varianzas iguales.

Fuente: elaboración propia en base a datos del Censo Nacional de población, hogares y viviendas. 2010, INDEC.

\section{DISCUSIÓN}

Aquellos departamentos del NOA que muestran mayor riesgo para la mortalidad infantil (tanto neonatal como pos-neonatal) han mostrado situaciones con deficiencias más profundas en relación a algunos determinantes sociales intermediarios de la salud. Entre ellos se destacan aspectos relacionados con la preparación y conservación de los alimentos y el hecho de tener electricidad, lo cual limita la tenencia de algunos artefactos eléctricos de gran utilidad, no sólo para esa conservación de alimentos, sino también para las comunicaciones y el acceso a la información a través de la computadora y el teléfono celular. También, se detectó como un DSS de mayor deficiencia en las zonas con alto riesgo para la MI (en sus dos componentes), el bajo nivel de instrucción de la población general, baja cobertura de salud y mayor número promedio de hijos nacidos vivos.

A su vez, se evidencia que no siempre coinciden los DSS que mostraron diferencias entre las zonas (de alto riesgo versus menor riesgo) de mortalidad reducible neonatal y de mortalidad pos-neonatal. Es decir, la diferencia estadísticamente significativa fue hallada entre las medias del porcentaje de viviendas con saneamiento ambiental 
deficiente y en el porcentaje de viviendas que reside en zona rural dispersa en la MI neonatal, pero no resultó significativa en la MI pos-neonatal. Esto se puede explicar por las características del tipo estudio ecológico ya que al considerar el conjunto de los individuos pueden estar ocultándose la heterogeneidad de una unidad geográfica considerada. La heterogeneidad, ya sea tanto de la mortalidad como de los determinantes sociales al interior de la unidad geográfica seleccionada, puede disimularse en el agrupamiento de los datos. De manera tal que los niños que fallecen en el periodo pos-neonatal en ese territorio pueden, individualmente, vivir en un ambiente de riesgo tal como lo muestra la evidencia científica y, por lo tanto, no resultar significativo ya que el resto del territorio tiene mejores indicadores de saneamiento ambiental. Por su parte, los DSS, que presentaron diferencias estadísticamente significativas en la MI pos-neonatal fueron la tenencia de heladera y la baja calidad de los materiales de la vivienda.

Los determinantes relacionados con las condiciones de la vivienda, el saneamiento y la alimentación también se detectaron como aspectos críticos en otros estudios que abordan la pobreza infantil( ${ }^{(24)}$.

Cabe resaltar que aun teniendo en cuenta las limitaciones del tipo de estudio y las técnicas aplicadas en esta investigación, no deja de ser otra evidencia científica más que sostiene que la mortalidad infantil reducible -tanto neonatal como pos-neonatalse asocia con condiciones de carencia más pronunciadas. Estos hallazgos son coincidentes con otras investigaciones que ha utilizado las mismas fuentes de datos pero diferentes técnicas de análisis como las realizadas por Bertone ${ }^{(8)}$, Herrero y Bosio $^{(18)}$, entre otros. A su vez, cabe aclarar que las características (tanto del nivel individual como familiar y poblacional) influyen entre sí, aspecto identificado en otros estudios sobre la pobreza multidimensional infantil ${ }^{(24)}$. Dicha influencia o sinergia actúan modificando su efecto sobre la reducibilidad de la mortalidad infantil ${ }^{(9)}$. La particularidad del tipo de estudio que se presenta, con información por conglomerado, permite hacer inferencias respecto al conjunto, lo que las decisiones individuales pueden influir e interactuar con los determinantes del conjunto de la población que se analiza en la mortalidad infantil no están contempladas. Las evidencias muestran que estas situaciones de carencia que se reflejan en los DSS se asocian con la mortalidad. Por ello aquellas políticas tendientes a mejorar las condiciones de vida de las personas en términos de educación, vivienda, seguridad alimentaria, acceso a la salud y conectividad, entre otras, también tienen a reducir la mortalidad infantil y garantizar el derecho a la vida de los niños y niñas del NOA.

\section{AGRADECIMIENTO}

Al Consejo Nacional de Investigaciones Científicas y Técnicas y a las Universidades Nacionales de Córdoba y de La Rioja.

\section{CONTRIBUCION DE LOS AUTORES:}

Todos los autores han contribuido conjuntamente $y$ en partes iguales en la elaboración del presente manuscrito.

\section{REFERENCIAS BIBLIOGRÁFICAS}

1. Finkelstein $\mathrm{JZ}$, Duhau $M$, Abeyá Gilardon E, Ferrario C, Speranza A, Asciutto C, et al. Mortalidad infantil en Argentina. $3^{a}$ revisión de los criterios de reducibilidad. Arch Argent Pediatr. 2015; 113(4): 352-8.

2. Mazzeo V. La mortalidad infantil en Argentina. Análisis de sus cambios y de las diferencias regionales. Población y Desarrollo-Argonautas y caminantes. 2014; 10: 9-2.

3. Mazzeo V. La situación de la saludenfermedad-atención de la primera infancia en la Ciudad de Buenos Aires 
Buenos Aires: Comisión sobre los Determinantes Sociales de la Salud; 2009.

6. Vega $A L$, Torcida $S$, Velázquez $\mathrm{GA}$. Análisis de la Evolución de la Tasa de Mortalidad Infantil en los Departamentos de Argentina. 19942003. Salud Colectiva. 2006 Septiembre - Diciembre; 2(3): 237-47.

7. Abriata MG, Fandiño ME. Abordaje de la mortalidad infantil en Argentina desde la perspectiva de las desigualdades en salud. Revista Argentina de Salud Pública. 2010 Marzo; 1(2): 43-5.

8. Bertone CL. Determinantes Sociales de la Mortalidad Infantil en Argentina 2000-2010. 2014.

9. Herrero $M$, Bossio J. Determinantes sociales de la mortalidad infantil por causas reducibles en la Argentina, 2009-2011. Población y Salud en Mesoamérica. 2017; 15(1): 1-29.

10. Solar O, Irwin A. A conceptual framework for action on the social determinants of health. Social Determinants of Health Discussion Paper 2 (Policy and Practice). Discussion paper (Policy and Practice). Geneva: World Health Organization, Commission on Social Determinants of Health; 2010. Report No.: ISBN 97892 41500852.

11. Irwin LG, Siddiqi A, Hertzman C. Desarrollo de la Primera Infancia: Un Potente Ecualizador. Informe Final para la Comisión sobre los Determinantes Sociales de la Salud de la Organización Mundial de la Salud. OMS, Comisión de los Determinantes Sociales de la Salud; 2007.

12. Naciones Unidas. Pobreza infantil en América Latina y el Caribe.: CEPALCELADE-UNICEF; 2010.

13. Domínguez L. El intervalo intergenésico: un factor de riesgo para complicaciones obstétricas y neonatales. Clínica e Investigación en Ginecología y Obstetricia. 2005; 32(03): 122.

14. UNFPA. Motherhood in Childhood: Facing the challenge of adolescent pregnancy. State of World Population 2013. 2013.

15. Castiel LD, Sánz Valero J. El acceso a la información como determinante social de la salud. Nutrición Hospitalaria. 2010: 26-30.

16. Chaparro Mendivelso J. Identificación de la segregación digital territorial en Bogotá, Colombia, a partir de la Encuesta de Calidad de Vida, 2007. Cuadernos de Geografía - Revista Colombiana. 2010;(19): p. 111-24.

17. Escuela Nacional de Sanidad (ENS). Método Epidemiológico Madrid: Instituto de Salud Carlos III - Ministerio de Ciencia e Innovación.; 2009.

18. Herrero MB, Bossio JC. Determinantes sociales de la mortalidad infantil por causas. Población y Salud en Mesoamérica. 2017; 15(9): p. 1-31.

19. Ministerio de Salud de la Nación. Mortalidad Infantil según Criterios de Reducibilidad. Serie 3- Número 56. Buenos Aires: Dirección de Estadísticas de Salud - Comisión Nacional de Clasificación de Enfermedades Dirección de Maternidad e Infancia; 2014. Report No.: ISSN 0325-0490.

20. Kulldorff M. A spatial scan statistic. Communications in Statistics: Theory and Methods. 1997; 26: p. 1481-96.

21. Kulldorf M. SaTScan ${ }^{\mathrm{TM}}$. [Online]; 2010 [cited 201306 10. Available from: https://www.satscan.org/cgibin/satscan/register.pl/Current\%20Vers ion:\%20SaTScan\%20v9.1.1\%20release d\%20March\%209\%202011.?todo=proc ess userguide download

22. Fernández M, Guevel C, KH, Marconi É, Massa C. Omisión de registro de nacimientos y muertes infantiles: magnitud, desigualdades y causas. Primera ed. Buenos Aires: Organización Panamericana de la Salud - Ministerio de Salud; 2008.

23. Ministerio de Salud. Registro de nacimientos en Argentina. Un estudio sobre la cobertura legal y estadística. Buenos Aires: 2017.

24. Tuñón I, Poy S, Coll A. La pobreza infantil en clave de derechos humanos y sociales. Definiciones, estimaciones y principales determinantes (2010-2014). Población \& Sociedad. 2016; 24(1): 101-33.

25. Wagstaff A. Desigualdades socioeconómicas y mortalidad infantil: comparación de nueve países en desarrollo. Bulletin of the World Health Organization. 2000; 78(1): 19-29.

26. Behm H. Determinantes Económicos y Sociales de la Mortalidad en América Latina. Salud Colectiva. 2011 MayoAgosto; 7(2): p. 231-53.

27. Benavides RD. Exploración de los Determinantes Sociales de la Salud en América Central: un análisis de Nicaragua, Guatemala, Honduras y El Salvador. Informe Estado de la Región 2008. 2008.

28. López Pardo CM. La medición del estado de salud de la población y su relación con los determinantes. Rev. cub. salud pública [online]. 2007; 33(1): p. 0-0.

29. Etchegoyen G, Paganini J. La relación entre los factores socioeconómicos y los programas sanitarios de salud maternoinfantil en 13 provincias 
argentinas. Rev Panam Salud Pública. 2007; 21(4): p. 223-30.

30. Buchbinder M. Mortalidad infantil y desigualdad socioeconómica en la Argentina. Tendencia temporal. Arch Argent Pediatr. 2008; 106(3): p. 212-8.

31. Longhi F. HOLOGRAMATICA - Facultad de Ciencias Sociales - UNLZ -. 2011; 3(14): p. 51-69.

32. Terrasa S, Rubinstein F, Giraudo N, Bortman M. Mortalidad infantil $y$ prematura en Argentina: poder explicativo de las diferencias y de acceso a servicios de salud. Archivos de Medicina Familiar y General. 2005; $1(2)$.

33. Castiel LDD, Sanz-Valero J. El acceso a la información como determinante social de la salud. Nutrición Hospitalaria. 2010; 3: p. 26-30.

34. Acosta L, Bertone C, Peláez E. Mortalidad y Años de Esperanza de Vida Perdidos a causa del consumo de alcohol en Argentina. 2008. Población y salud en Mesoamérica. 2012; 9(2): p. 1-21.

35. Arriaga EE. Los años de vida perdidos: su utilización para medir los niveles y cambios de la mortalidad. Notas de Población. 1996 Junio; 24(63): p. 7-38.

36. World Health Organization. Global status report on alcohol and health 2018. Switzerland: 2018. Report No.: 978-92-4-156563-9.

37. Santamaría Ulloa C. Análisis para la determinación de conglomerados espacio - temporales en la incidencia de cáncer. Costa Rica, 1990-1997. 2002. Trabajo final de graduación presentado a la Escuela de Estadística, para optar por el grado de Máster en Población y Salud.

38. Ministerio de Salud de la Nación. Definiciones y Conceptos en estadísticas de salud. [Online]; s/f [cited 201310 15]. Available from:

https://www.argentina.gob.ar/salud/dei s/ses/definiciones

39. Naciones Unidas. Principales cambios en las boletas de los censos latinoamericanos de las décadas de 1990, 2000 y 2010. Serie-Manuales. Santiago de Chile: Centro Latinoamericano y Caribeño de Demografía, División de Población de la Comisión Económica para América Latina y el Caribe (CEPAL); 2013. Report No.: ISSN 1680-886X.

40. UNICEF. El derecho al registro universal y oportuno de niñas y niños. Avances y desafíos para la normativa legal y las estadísticas vitales. Primera ed. Buenos Aires: Fondo de las Naciones Unidas para la Infancia (UNICEF); 2013.

41. Ministerio de Salud de la Nación. Mortalidad Infantil según Criterios de Reducibilidad. Serie 3- Número 56. Buenos Aires, Dirección de Estadísticas de Salud - Comisión Nacional de Clasificación de Enfermedades Dirección de Maternidad e Infancia; 2014. Report No.: ISSN 0325-0490. 